\title{
Estimation and Reduction of Target Registration Error
}

\author{
Ryan D. Datteri and Benoît M. Dawant \\ Department of Electrical Engineering and Computer Science, \\ Vanderbilt University, Nashville, TN 37235, USA \\ \{ryan.d.datteri, benoit. dawant\} @vanderbilt.edu
}

\begin{abstract}
Fiducial-based registration is often utilized in image guided surgery because of its simplicity and speed. The assessment of target registration error when using this technique, however, is difficult. Although the distribution of the target registration error can be estimated given the fiducial configuration and an estimation of the fiducial localization error, the target registration error for a specific registration is uncorrelated with the fiducial registration error. Fiducial registration error is thus an unreliable predictor of the target registration error for a particular case. In this work, we present a new method to estimate the quality of a fiducial-based registration and show that our measure is correlated to the target registration error and that it can be used to reduce registration error caused by fiducial localization error. This has direct implication on the attainable accuracy of fiducial-based registration methods.
\end{abstract}

Keywords: Image registration, registration circuits, rigid registration, fiducial registration, image guided surgery, registration error.

\section{Introduction}

Fiducial-based registration is an important technique in Image Guided Surgery (IGS). It is often utilized to align image information to the surgical space in an operating room. In this context, fiducial markers are attached to the patient and an image is acquired. The physical location of the fiducial markers in the operating room is obtained as well as the location of the markers in the image and the two point sets (fiducial configurations) are registered to each other. Error in identifying the correct location of the individual fiducials, called Fiducial Localization Error (FLE) [1] may occur which causes error in the registration between the image and surgical space. An analytical solution for the distribution of errors in fiducial registration has been proposed [1, 2, 3], but this solution does not permit the assessment of the target registration error in a particular case. Fiducial Registration Error (FRE) is often used as a surrogate for the Target Registration Error (TRE) that is the quantity of clinical interest [4]. Unfortunately, it has been shown [5] that TRE and FRE are uncorrelated. FRE is thus an unreliable predictor of registration accuracy.

In this work, we propose a method to estimate the quality of a registration that correlates with TRE and therefore produces a value that correlates with the true registration accuracy at a target location. The next section describes our technique, which we call AQUIRC for Assessing Quality Using Image Registration Circuits. In 
the results section, simulation results we have produced to demonstrate the correlation between our measure and TRE are presented and future work and applications are discussed.

\section{General Algorithm}

This algorithm was first proposed in [6] for global atlas selection and was utilized to estimate the quality of intensity-based rigid image registration in [7]. Briefly, AQUIRC builds on the idea of registration circuits which was proposed as a consistency measure by Woods et al. [8] and Holden et al. [9]. Here, a registration circuit involves three fiducial configurations $\mathrm{A}, \mathrm{B}$, and $\mathrm{C}$ and three transformations $T_{\mathrm{AB}}, T_{\mathrm{BC}}$, and $T_{\mathrm{CA}}$. The configurations' coordinates differ by the assumed localization error of the individual fiducials. As discussed by Fitzpatrick [10], using only one

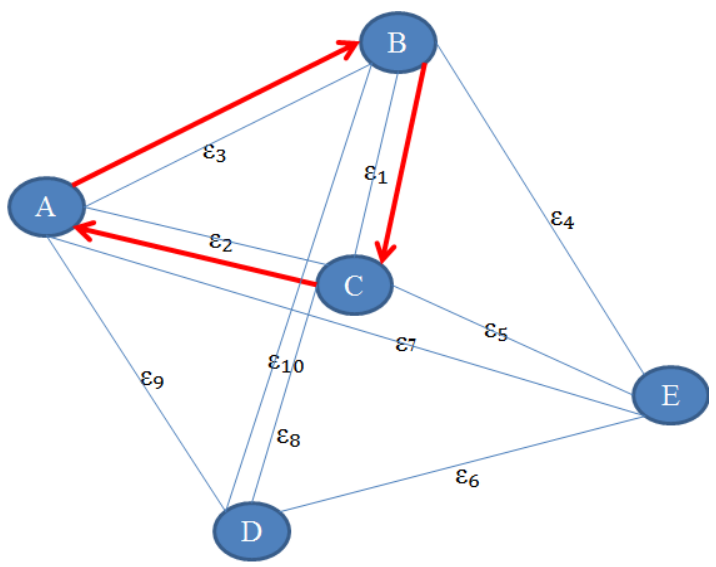

Fig. 1. Example complete graph with one circuit shown in red arrows registration circuit can lead to an underestimation of registration error because the error made along one edge in the circuit may correct error introduced from a separate edge in the circuit.

In this work, we expand upon the idea of a registration circuit to multiple circuits. We start with a set of fiducial configurations and compute pair-wise registrations between all elements in the set, creating a complete graph as shown in Figure 1. The complete graph of registrations is similar to what is done by Christensen [11]. In [11], however, the set consisted of medical images and the complete graph was used as an overall measure of quality for a registration algorithm, rather than as a method to determine the quality of individual registrations as we have done here. If our initial set contains $\mathrm{N}$ fiducial configurations (i.e., the same set of fiducial markers but with the position perturbed by the assumed fiducial localization error) the graph contains $\left(\begin{array}{l}\mathrm{N} \\ 2\end{array}\right)$ edges. With each edge in this graph, we associate an initially unknown measure of registration quality called $\varepsilon$ that we wish to solve for. There are $\left(\begin{array}{l}\mathrm{N} \\ 3\end{array}\right)$ unique registration circuits that can be formed from a complete graph (we have used registration circuits of size 3; the circuit size can be increased to form more registration circuits but this was not explored here).

Next, we define a measure of registration error that can be computed across a circuit. Here, to compute this error, we select a target point or set of points in A, say X. We then compute the transformed point(s) $\mathrm{X}^{\prime}$ as $\mathrm{X}^{\prime}=\mathrm{T}_{\mathrm{AB}}\left(\mathrm{T}_{\mathrm{BC}}\left(\mathrm{T}_{\mathrm{CA}}(\mathrm{X})\right)\right.$ ). We note the important fact that the order in which transformations are composed is critical and that this order differs from the originally proposed registration circuit in $[8,9]$. The quality of 
registrations across circuit $\mathrm{A}, \mathrm{B}, \mathrm{C}$, is then defined as $\mathrm{E}_{\mathrm{C}}=\operatorname{dissimilarity}\left(\mathrm{X}, \mathrm{X}^{\prime}\right)$. The value, $E_{C}$, is affected by the error of three registrations, i.e., the registration error between $\mathrm{A}$ and $\mathrm{B}$, the registration error between $\mathrm{B}$ and $\mathrm{C}$, and the registration error between $\mathrm{C}$ and A. With only one circuit the contribution of each component cannot be computed. It can, however, be estimated with more than one circuit. To achieve this we make the assumption that each registration affects the quality measure multiplicatively, i.e., $\varepsilon_{\mathrm{ABC}}=$ $\varepsilon_{\mathrm{A}} * \varepsilon_{\mathrm{B}} * \varepsilon_{\mathrm{C}}$ or, $\log \left(\varepsilon_{\mathrm{ABC}}\right)=\log \left(\varepsilon_{\mathrm{A}}\right)+\log \left(\varepsilon_{\mathrm{B}}\right)+\log \left(\varepsilon_{\mathrm{C}}\right)$. An additive model may also be applicable but was not tested in this work. Computing this expression for all possible circuits and rearranging them in matrix form, we obtain

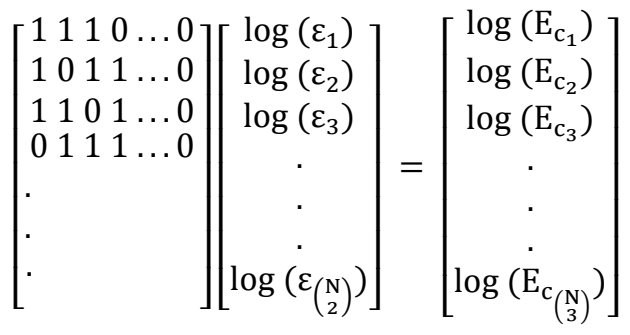

in which $\mathrm{E}_{\mathrm{C}_{\mathrm{i}}}$ is defined as the dissimilarity $\left(\mathrm{X}, \mathrm{X}^{\prime}\right)$ value around circuit $\mathrm{i}$. This expression can be rewritten as $\overline{\bar{P}} \log (\bar{\varepsilon})=\log \left(\overline{\mathrm{E}}_{\mathrm{c}}\right)$. As a result of the multiplicative assumption, $\log (\bar{\varepsilon})$ can be solved for using a linear least squares solution

$$
\log (\bar{\varepsilon})=\left(\overline{\bar{P}}^{\mathrm{T}} \overline{\overline{\mathrm{P}}}\right)^{-1} \overline{\overline{\mathrm{P}}}^{\mathrm{T}} \log \left(\overline{\mathrm{E}_{\mathrm{c}}}\right)
$$

and finally solving for $\bar{\varepsilon}$

$$
\bar{\varepsilon}=\mathrm{e}^{\log (\bar{\varepsilon})}
$$

We are currently working on a proof of conditions on the registration circuits for when $\overline{\overline{\mathrm{P}}}$ is full rank and therefore $\left(\overline{\overline{\mathrm{P}}}^{\mathrm{T}} \overline{\overline{\mathrm{P}}}\right)$ is invertible. Experimentally $\overline{\overline{\mathrm{P}}}$ has been observed to be full rank when $N \geq 5$. We define $\overline{\bar{P}}$ to be all unique circuits in the graph of size 3 .

There are multiple ways to define the circuits that are utilized to create the $\overline{\bar{P}}$ matrix. In this work, we utilize the set of unique circuits of size 3 in the graph. For example, for the three nodes $\mathrm{A}, \mathrm{B}$, and $\mathrm{C}$ in Figure 1, we consider only one circuit e.g., $\mathrm{T}_{\mathrm{AB}}\left(\mathrm{T}_{\mathrm{BC}}\left(\mathrm{T}_{\mathrm{CA}}\right)\right)$.

\subsection{AQUIRC Applied to Fiducial-Based Registration}

For this work, we utilize the fiducial registration method that minimizes the FRE between two sets of points. This fiducial registration method is standard and uses singular-value decomposition as proposed by Arun et al [12]. We also define the function dissimilarity $\left(\mathrm{X}, \mathrm{X}^{\prime}\right)$ to be the $\operatorname{TRE}\left(\mathrm{X}, \mathrm{X}^{\prime}\right)$ where $\operatorname{TRE}\left(\mathrm{X}, \mathrm{X}^{\prime}\right)$ is defined as the Euclidean distance between $\mathrm{X}$ and $\mathrm{X}^{\prime}$ because, as explained below, our set $\mathrm{X}$ 
contains a single element. In this particular study, we define two spaces: the image space and the surgical space, to mimic the situation of a typical point-based registration problem where pre-operative images need to be registered to the patient in the operating room. We define $\mathrm{X}$ as the target point and we select that point in image space. As discussed below, we introduce a known transformation between image and surgical space that can be large and we perturb the position of the fiducials in image space.

\section{Experiments and Results}

To test our algorithm we repeat an experiment that was performed by Fitzpatrick which showed analytically and experimentally that FRE and TRE are uncorrelated [5]. Using the same experiment we show that our quality measure is correlated to the TRE.

\subsection{Experiments}

In Experiment 1 in [5], Fitzpatrick simulates an actual Deep Brain Stimulation case with 4 fiducials and a target location in the deep brain. The location of the four fiducials $x_{1}, x_{2}, x_{3}$, and $x_{4}$ as well as the target position $x_{t}$ are:

$$
x_{1}=\left[\begin{array}{l}
197 \\
217 \\
115
\end{array}\right], \quad x_{2}=\left[\begin{array}{l}
109 \\
225 \\
121
\end{array}\right], \quad x_{3}=\left[\begin{array}{c}
83 \\
139 \\
127
\end{array}\right], \quad x_{4}=\left[\begin{array}{l}
202 \\
132 \\
130
\end{array}\right], \quad x_{t}=\left[\begin{array}{c}
144 \\
155 \\
57
\end{array}\right]
$$

Following the same simulation model as in [5], we first apply a rotation $R$ and translation $t$ to the location of the fiducials $x_{1}, x_{2}, x_{3}$, and $x_{4}$ as well as to the target position $x_{t}$, which results in the corresponding positions $y_{1}, y_{2}, y_{3}, y_{4}$, and $y_{t}$. We consider the fiducials $x_{1}, x_{2}, x_{3}$, and $x_{4}$ and $x_{t}$ to be in the image space while the rotated and translated fiducials $y_{1}, y_{2}, y_{3}, y_{4}$, and $y_{t}$ are considered to be in the surgical space. Again, as in [5], we set the rotation $R$ to be 10, 20, and -30 degrees about the $x, y$, and $z$ axes and we set the translation $t$ to be $(7,-10,100) \mathrm{mm}$ (which was chosen as an arbitrary mis-registration in [5]).

We then perturb the location of the fiducials in image space using a fiducial localization error drawn from a random distribution with zero mean and variance of FLE/3, where FLE is set to $1 \mathrm{~mm}$. This is done N-1 times to create a set of N-1 perturbed fiducial configurations (as seen in the left of Figure 2). We then compute all pair-wise fiducial registrations between each of the N-1 fiducial configurations in the image space as well as between these and the unperturbed rotated fiducial configuration in the surgical space, creating the complete graph of registrations necessary to run our algorithm which we use to calculate the $\varepsilon$ value for each of the registrations. There are three quantities of importance in this work: TRE, FRE, and $\varepsilon$. These three values are calculated for the registrations between the fiducial configurations in the image space and the fiducial configuration in the surgical space, which results in $\mathrm{N}-1$ values for TRE, FRE, and $\varepsilon$ (this is represented as the red links in Figure 2). FRE is defined as the root mean square distance between the fiducial points 
in image space and the points transformed from image space to surgical space using the computed transformations. For each run, we thus produce N-1 FRE values. Similarly, TRE is defined as the Euclidean distance between the target position in surgical space and the position of the target transformed from image space to surgical space. We repeat this process in a Monte Carlo simulation; creating thousands of image space fiducial configurations with randomized FLEs.

In the first experiment we utilize an $\mathrm{N}$ of size 30 over 1000 simulations. We calculate the correlation between the TRE values and $\varepsilon$ values and the FRE and $\varepsilon$ values. In the second experiment we test the effect $\mathrm{N}$ has on our results by varying the size of $\mathrm{N}$ from 5 to 25 using 5000 simulations for each value of N. Again, we calculate the correlation between TRE and the $\varepsilon$ values for each value of N. Finally, in the third experiment, we test the ability of our algorithm to improve the TRE in a fiducial-based registration scenario. To do this we use an $\mathrm{N}$ of 30 over 1000 simulations. For each simulation we consider the registration between the 29 image space fiducials and the surgical space fiducial and for all 29 registrations calculate the mean TRE, the min TRE, the max TRE, the TRE of the fiducial configuration that AQUIRC identifies as being of the highest quality, and the TRE of the fiducial configuration with the minimum FRE.

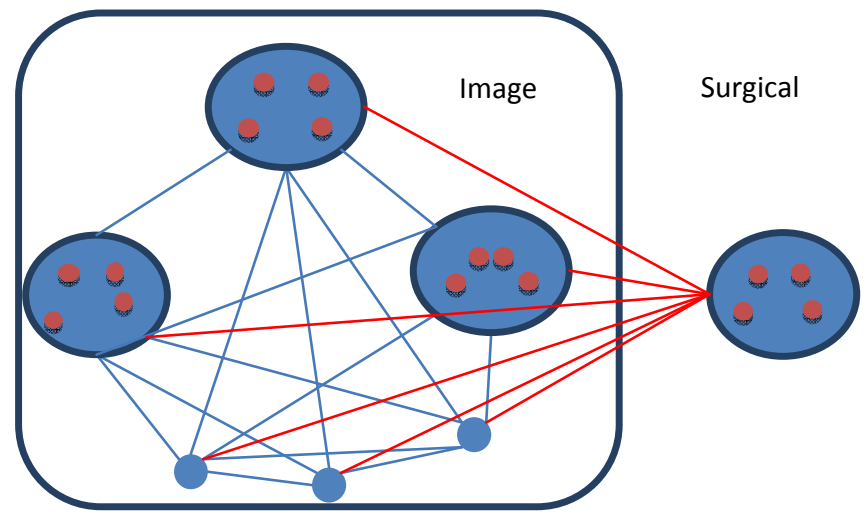

Fig. 2. Diagram of the experiment methodology. The image space contains N-1 fiducial configurations that are created by adding FLE to the original fiducial locations. The surgical space contains the rotated and translated fiducials. Each set of fiducial configurations are registered to every other configuration, both in image space and in surgical space. The red links represent the registrations for which we calculate the TRE, FRE and $\varepsilon$ values.

\subsection{Results}

The results of experiment 1 are shown in Figures 3. In the left of Figure 3 we show a scatter plot of the FRE and TRE values (the points in both scatter plots were reduced to a random sampling of 1000 data points for better visualization). As can be seen, we produce a correlation that is very similar to the one found in [5], with an $r=-0.0012$, which is not statistically significant $(\mathrm{p}=0.8351)$. In the right of Figure 3 we show the correlation between our algorithm's $\varepsilon$ value and TRE. In this case there is a statistically significant correlation of $\mathrm{r}=0.6086$ and a $\mathrm{p}<0.001$. The results of 
experiment 2 are shown in Figure 4. By increasing the number of fiducial configurations that we utilize in the image space we can increase the correlation between TRE and $\varepsilon$, producing better estimations of the quality of error in fiducial registrations. The results of experiment 3 are shown in Figure 5; it shows the practical utility of our algorithm. If, for every simulation, we choose the configuration with the lowest $\varepsilon$ value, the TRE is reduced by a statistically significant $0.1347 \mathrm{~mm}$ when compared to the mean TRE value and is reduced by a statistically significant 0.1367 when compared to the TRE of the fiducial configuration with the minimum FRE. The figure also shows the mean and standard deviation of the TRE values when the configurations with the max and min TRE values are selected at every run.
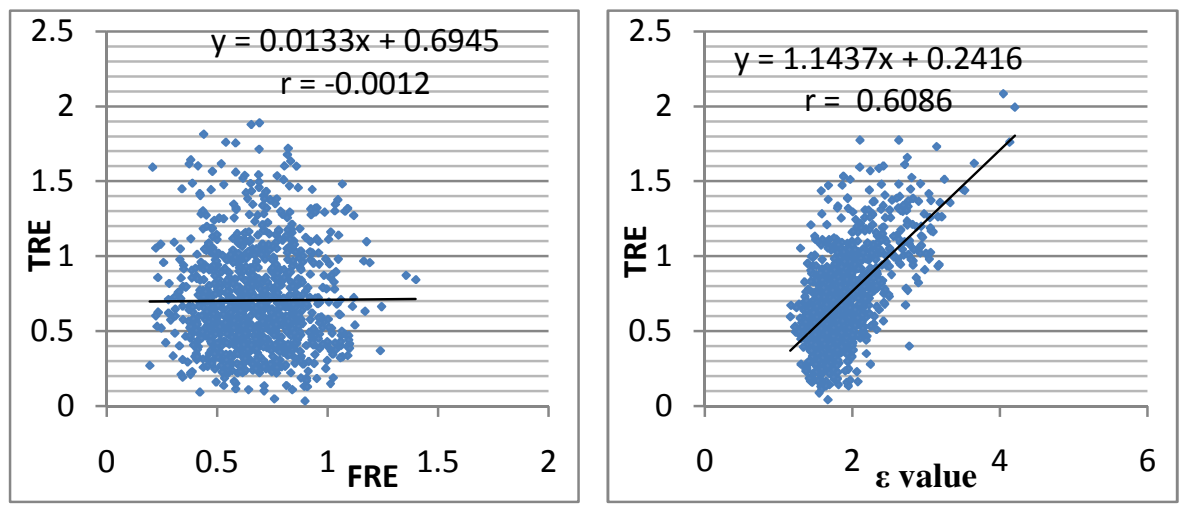

Fig. 3. Left: Scatter plot of the TRE and FRE values. Right: Scatter plot of the TRE and $\varepsilon$ values.

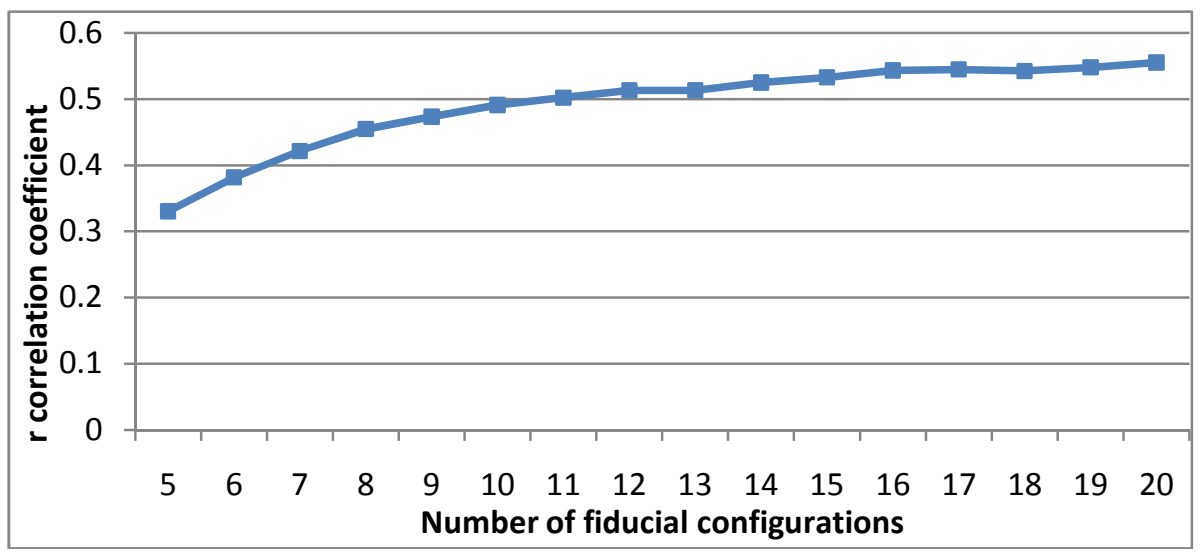

Fig. 4. Correlation between TRE and $\varepsilon$ as a function of the number of fiducial configurations utilized in the surgical space. 


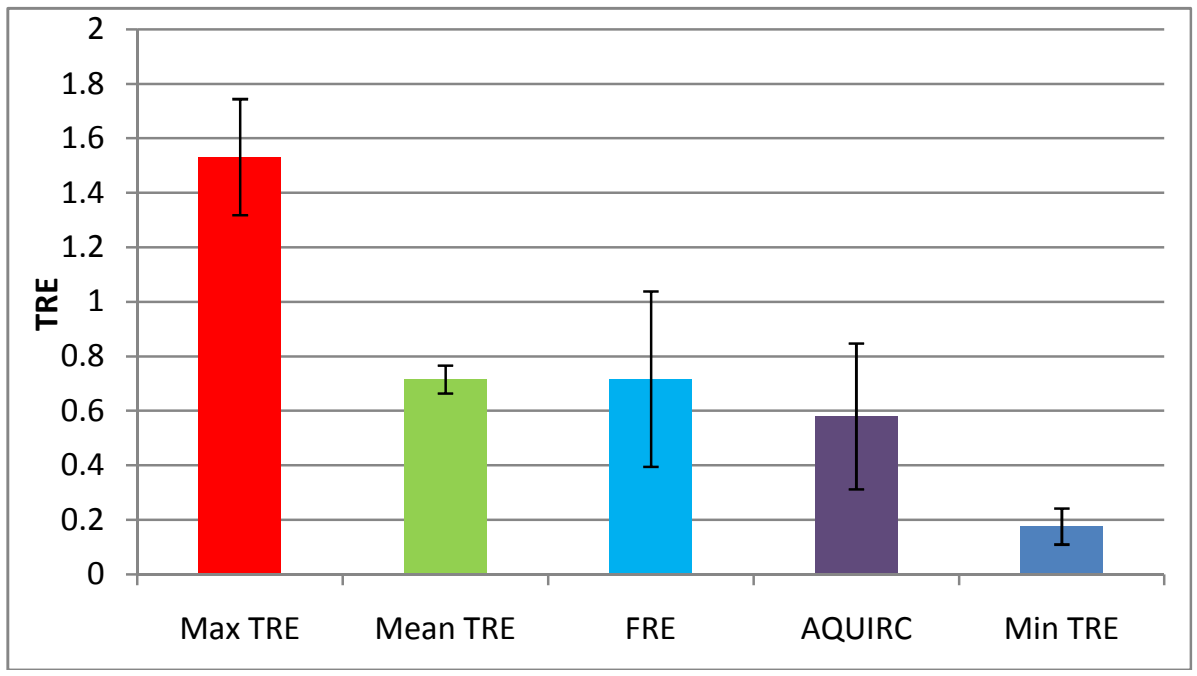

Fig. 5. Bar graph of the mean value and standard deviation of the TRE value across 1000 simulations. For each simulation we calculate the mean TRE of all 30 fiducial configurations, the min TRE of all 30 fiducial configurations, the max TRE of all 30 fiducial configurations, the TRE of the fiducial configuration that AQUIRC identifies as being of the highest quality, and TRE of the fiducial configuration with the minimum FRE.

\section{Discussions/Future Work}

In this work we have introduced a method that produces a measure of registration quality that is correlated to the target registration error. We are unaware of any other published work that describes a technique that is able to do so. We have shown that the number of fiducial configurations that are utilized in the complete graph of registrations affects the quality of our algorithm's estimation and the correlation between our measure and TRE increases as the number of configuration increases. Most importantly for practical applications, we also show that by choosing the configuration that our algorithm identifies as producing the best registration, we can reduce the average TRE.

To use our algorithm in practice, all that is needed is to acquire the location of the fiducial markers in the image space as well as in surgical space. The location of the markers in image space can then be randomly perturbed by an FLE that is representative of the error that naturally occurs when attempting to identify the coordinates of the markers (alternatively, the position of the markers in surgical space could be perturbed). These sets of markers can then be registered together to form a complete graph of registrations and AQUIRC is applied. The $\varepsilon$ value of the unperturbed configuration, i.e., the position of the fiducials selected by the end user, can then be compared to the distribution of $\varepsilon$ values produced by our algorithm. If the unperturbed $\varepsilon$ value is large compared to the perturbed $\varepsilon$ values, the end user could be warned of a potential registration problem. 
In the future, we will investigate various types of models that could better represent the combination of error that occurs when combining multiple transformations than the multiplicative model we have used in this work. We will also explore further the distribution of $\varepsilon$ values and attempt to define statistical tests that would permit to quantify the quality of a particular registration.

As discussed earlier, the order in which transformations are composed when computing the registration error across a circuit is important. In fact, if $\mathrm{X}^{\prime}$ is computed as $\mathrm{X}^{\prime}=\mathrm{T}_{\mathrm{AB}}\left(\mathrm{T}_{\mathrm{BC}}\left(\mathrm{T}_{\mathrm{CA}}(\mathrm{X})\right)\right.$ ), we have not observed a correlation between the TRE and $\varepsilon$ values. The theoretical reasons for this observation have not yet been elucidated and are under investigation. If successful, this algorithm would provide end users with quantitative measures of accuracy for a particular registration. This would represent a major advance in the field of fiducial-based registration.

Acknowledgement. This work was partially supported by NSF grant DMS-0334769 and NIH Grant No. R01EB006193.

\section{References}

1. Fitzpatrick, J.M., West, J.B., Maurer Jr., C.R.: Predicting error in rigid-body point-based registration. IEEE Transactions on Medical Imaging 17, 694-702 (1998)

2. Fitzpatrick, J.M., West, J.B.: The distribution of target registration error in rigid-body point-based registration. IEEE Transactions on Medical Imaging 20, 917-927 (2001)

3. Danilchenko, A., Fitzpatrick, J.M.: General approach to first-order error prediction in rigid point registration. IEEE Trans. Med. Imaging 30, 679-693 (2010)

4. Suess, O., Picht, T., Kuehn, B., Mularski, S., Brock, M., Kombos, T.: Neurosurgery without rigid pin fixation of the head in left frontotemporal tumor surgery with intraoperative speech mapping. Operative Neurosurgery 60(suppl. 2), 330-338 (2007)

5. Fitzpatrick, J.M.: Fiducial registration error target registration error are uncorrelated. In: SPIE. Medical Imaging: Visualization, Image-Guided Procedures, and Modeling, vol. 7261(1), pp. 1-12 (2009)

6. Datteri, R.D., Asman, A.J., Landman, B.A., Dawant, B.M.: Estimation of Registration Quality Applied to Multi-Atlas Segmentation. In: MICCAI (2011)

7. Datteri, R.D., Dawant, B.M.: Estimation of Rigid-Body Registration Quality Using Registration Networks. In: Proc. SPIE, Medical Imaging 2011: Image Processing (2011)

8. Woods, R.P., Grafton, S.T., Holmes, C.J., Cherry, S.R., Mazziotta, J.C.: Automated image registration: I. General methods and intrasubject, intramodality validation. J. Comput. Assist. Tomogr. 22, 139-152 (1998)

9. Holden, M., Hill, D.L.G., Denton, E.R.E., Jarosz, J.M., Cox, T.C.S., Rohlfing, T., Goodey, J., Hawkes, D.J.: Voxel similarity measures for 3D serial MR brain image registration. IEEE Transactions on Medical Imaging 19, 94-102 (2000)

10. Fitzpatrick, J.M.: Detecting failure, assessing success. In: Hajnal, J.V., Hill, D.L.G., Hawkes, D.J. (eds.) Medical Image Registration, pp. 117-139. CRC Press, Florida (2001)

11. Christensen, G.E., Johnson, H.J.: Invertibility and transitivity analysis for nonrigid image registration. J. Electron. Imaging 12, 106-117 (2003)

12. Arun, K.S., Huang, T.S., Blostein, S.D.: Least-Squares Fitting of two 3-D Point Sets. IEEE Trans. Pattern Anal. 9(5), 698-700 (1987) 